\title{
Lumen
}

Selected Proceedings from the Canadian Society for Eighteenth-Century Studies

\section{La réception de l'opéra par les Philosophes}

\section{Béatrice Didier}

Volume 17, 1998

Theatre of the world

Théâtre du monde

URI : https://id.erudit.org/iderudit/1012378ar

DOI : https://doi.org/10.7202/1012378ar

Aller au sommaire du numéro

Éditeur(s)

Canadian Society for Eighteenth-Century Studies / Société canadienne d'étude du dix-huitième siècle

ISSN

1209-3696 (imprimé)

1927-8284 (numérique)

Découvrir la revue

Citer cet article

Didier, B. (1998). La réception de l'opéra par les Philosophes. Lumen, 17, 1-10. https://doi.org/10.7202/1012378ar

Copyright (c) Canadian Society for Eighteenth-Century Studies / Sociéte canadienne d'étude du dix-huitième siècle, 1998
Ce document est protégé par la loi sur le droit d'auteur. L'utilisation des services d'Érudit (y compris la reproduction) est assujettie à sa politique d'utilisation que vous pouvez consulter en ligne.

https://apropos.erudit.org/fr/usagers/politique-dutilisation/ 


\section{La réception de l'opéra par les Philosophes}

Dans la vie sociale du XVIIIème siècle, l'Académie royale de musique tient une place importante. Les écrivains sont nourris d'opéra: ils en entendent aussi chez des particuliers, nobles ou riches financiers, ainsi La Pouplinière qui eut une telle importance pour la carrière de Rameau. Les conversations des salons s'alimentent de discussions sur la musique; les diverses querelles musicales du siècle se cristallisent essentiellement autour de l'opéra. Le sujet quej'annonce risquerait donc d'être immense, et je l'ai déjà traité en grande partie dans La musique des Lumières (1985). Par rapport à ce livre, par rapport à tant d'études de musicologues, d'historiens de la musique et de la littérature qui avaient paru auparavant et qui se sont multipliées depuis, je risquerai donc de faire double emploi.

C'est pourquoi j'ai choisi de partir d'un aspect très limité et précis, mais qui permettra d'élargir la question ensuite et d'aborder, je l'espère, les problèmes essentiels que l'opéra a posés à l'esthétique et à la philosophie des Lumières. La représentation d'Hippolyte et Aricie, en 1733, a été une véritable révolution. C'est ce caractère en quelque sorte initial qui m'a attirée, puisque c'est la première oeuvre lyrique de Rameau. Par sa date même, l'opéra se situe nettement avant la Querelle des Bouffons qui amène un gauchissement, une simplification polémique de la réflexion des Lumières. On m'objectera peut-être que cette oeuvre ne représente qu'une des formes que prend alors le théâtre lyrique: il faudrait parler aussi de la pastorale, de la comédie-ballet, ou bien si l'on se situait plus tard dans le siècle, de l'opéra-comique. Je pense cependant que la tragédie en musique permet une réflexion assez large pour que les autres genres puissent être aussi abordés.

Rameau a attendu cinquante ans pour se risquer à une oeuvre lyrique; jusque-là il est connu par son Traité d'Harmonie (1722) et ses oeuvres pour clavecin; il est donc en quelque sorte un débutant; nous aurions tendance à penser qu'il pourrait collaborer avec un écrivain plus connu que l'abbé Pellegrin, ainsi avec Voltaire, puisque Samson, à la même époque est le fruit de cette collaboration d'un grand musicien et d'un grand écrivain. Mais Voltaire est de caractère difficile, Rameau aussi. Au total, Rameau 
préfère travailler avec ce polygraphe qu'est l'abbé Pellegrin. L'anecdote de la caution montre bien cependant qu'en 1733 l'échelle des notoriétés n'est pas du tout la nôtre: Rameau n'est pas connu comme auteur lyrique, c'est lui qui est demandeur et l'abbé, bien connu au contraire comme librettiste, qui hésite, jusqu'à ce qu'une audition chez la Pouplinière l'ait convaincu. Pellegrin (1663-1745) qui est alors largement l'aîné de Rameau (1683-1764) aurait écrit cinq cent mille vers au cours de son existence; poésies religieuses, livrets d'opéra, rien ne l'effraie.

Il est de coutume de déplorer la faiblesse du livret d'Hippolyte et Aricie: c'est peut-être mal comprendre les exigences du genre que de le comparer à la Phèdre de Racine. Quoiqu'il en soit, la distance qu'établit Pellegrin, par son livret et par les déclarations de sa préface, à l'endroit de ce modèle prestigieux et gênant, permettent de cerner assez bien quelles sont les questions essentielles que posent aux Philosophes la tragédie en musique, et plus généralement l'opéra. En effet la défense d'Hippolyte et Aricie qu'entreprend l'abbé Pellegrin dans sa préface, repose sur une tentative de définition générique: 'je n'aurais jamais osé, après un auteur tel que Racine, mettre une Phèdre au théâtre, si la différence du genre ne m'eût rassuré'. Outre la différence qui existe entre le génie de Racine et l'habileté de Pellegrin, deux différences profondes tiennent en effet à la nature même de la tragédie en musique. Si Pellegrin n'intitule pas son livret Phèdre, ce n'est pas seulement à cause de Racine, c'est aussi que l'angle de vue a complètement changé et qu'il ne peut pas considérer Phèdre comme le personnage-vedette. Si la tragédie au théâtre finit mal, la tragédie en musique, elle, connaît le plus souvent une fin heureuse. Il eût été scandaleux et contraire à la tradition, de supposer une fin heureuse à l'amour de Phèdre pour Hippolyte. Au contraire, les amours d'Hippolyte et d'Aricie, dans leur innocence et leur jeunesse pouvaient avoir une fin heureuse et aboutir à un mariage rassurant pour les moralistes, mais aussi bien conforme à l'esprit d'un genre où le caractère galant n'est jamais oublié.

Racine avait éliminé de son oeuvre, autant que faire se peut, le merveilleux condamné par les théoriciens de la tragédie; pourtant la tradition racontait que Thésée avait été aux Enfers, qu'Hippolyte périssait victime d'un monstre marin envoyé par Neptune. On sait comment le discours remplace l'action dans la tragédie classique. La célèbre tirade de Théramène permet à la fois de respecter l'unité de lieu et de ne pas montrer d'animaux mythiques sur la scène. Mais l'opéra n'est pas assujetti à cette double contrainte. L'acte second qui est d'ailleurs un des meilleurs moments de cet opéra, se passe aux Enfers. A la scène 3 de l'acte IV, le livret prévoit: 'La mer s'agite; on en voit sortir un monstre horrible'; 'Hippolyte sur son char s'avance vers le monstre; 'Le monstre blessé par Hippolyte, le couvre de feu et de fumée, tout se dissipe enfin, 
et l'on ne voit plus que le char brisé'. Dans sa préface, l'abbé Pellegrin se justifie: 'Je sais que l'unité de lieu n'est pas scrupuleusement observée dans cette tragédie, mais mon sujet était de nature à ne pouvoir se passer d'un privilège dont on ne doit pas contester la possession au genre lyrique'.

Néanmoins l'abbé Pellegrin ne se contente pas de cette justification générique du merveilleux. Sa préface avance aussi deux arguments qui dénotent malgré tout une certaine gêne de son temps devant le surnaturel: le merveilleux, prétend-il, dans son oeuvre, ne s'oppose pas au vraisemblable, mais au contraire le renforce. Chez Racine, en effet, Thésée paraît 'trop crédule'; il condamne 'un fils aussi vertueux qu'Hippolyte' 'sur la déposition d'une femme suspecte, et sur l'indice d'une épée qu'on pouvait avoir prise à son insu'. Le merveilleux permet de 'mieux fonder la condamnation d'Hippolyte'. Elle résultera de l'accomplissement du fatal oracle qu'ont proféré les Parques, avant de laisser Thésée sortir des Enfers. Spécieux plaidoyer: l'invraisemblance du merveilleux viendrait donc au secours de la vraisemblance psychologique menacée.

D'autre part le merveilleux doit avoir des limites, pense l'abbé Pellegrin, même à l'opéra. 'Je n'ai pas osé porter le merveilleux jusqu'à ressusciter Hippolyte'. Il est probable que l'abbé ne voulait pas toucher à une question-tabou, celle de la Résurrection; mais l'argument qu'il donne dans sa préface est curieux: il n'a pas voulu remettre en cause la hiérarchie des dieux: Diane ne devrait donc pas céder à l'Amour. Seul le Destin est supérieur à toutes les divinités païennes; et c'est lui l'arbitre qui finalement permet qu'Hippolyte soit vivant et épouse Aricie.

Faut-il faire de l'abbé Pellegrin un Philosophe? Dans sa vie, il ne s'est pas écarté de la discipline ecclésiastique; mais il est le reflet de son temps et sa parenté avec les Philosophes apparaît surtout à deux moments de ce livret. Aricie était vouée à Diane et au célibat. Au moment de prononcer des voeux qui rappellent incontestablement la prise de voile dans le culte catholique, elle s'insurge. Le choeur des prêtresses chante: 'Non, non, un coeur, forcé n'est pas digne des Dieux; / Le sacrifice en est un crime' (I, 4). L'abbé Pellegrin annonce-t-il la Religieuse de Diderot? En tout cas Diane émet un principe cher aux Lumières: 'On peut servir Diane avec le même zèle / Dans son temple et dans ses forêts' (I, 5). On peut adorer Dieu partout, et aussi bien dans la nature que dans un couvent. La suprématie du Destin à la fin de l'opéra reprend aussi un thème du déisme des Lumières: la diversité des cultes s'efface devant la suprématie d'un Dieu juste et équitable. 
La philosophie de l'abbé Pellegrin nous importerait cependant assez peu, si nous n'avions trouvé dans sa préface et dans le traitement qu'il fait subir à l'histoire de Phèdre les éléments essentiels de la réflexion esthétique des Philosophes devant l'opéra. On y lit d'abord une justification même de l'opéra. L'opéra a eu à défendre ses droits à l'existence, pratiquement dès sa naissance. Une tradition philosophique dont on trouve aussi bien les marques chez Saint-Evremond que chez Fontenelle, attaque l'opéra au nom de la vraisemblance. Est-il vraisemblable, en effet, que des dieux païens descendent sur la scène; que des personnages que l'on croyait morts, apparaissent brusquement comme vivants; est-il vraisemblable que l'on chante dans toutes les occasions, pour déclarer son amour ou sa haine, pour vivre ou pour mourir? Il existe une continuité entre les critiques inspirées par un respect étroit des règles de l'esthétique classique en matière de théâtre et le rationalisme des Lumières. Filiation d'autant plus facile à établir, qu'il n'y a pas eu chez les Philosophes de véritable révolution en matière esthétique, et que les plus audacieux en matière politique et religieuse ne cessent de proférer leur complète admiration envers l'art du siècle précédent. Comment concilier alors ces présupposés esthétiques et cette passion de l'opéra si ancrée dans leur sensibilité, si visible dans leur vie quotidienne? Après 1750 (pour simplifier), une issue à ce conflit sera proposée avec le développement d'un théâtre lyrique qui montrerait davantage la vie quotidienne. Inutile de faire descendre des dieux sur scène pour troubler la vie tranquille du Devin du village. Mais dans la première moitié du siècle, l'opéra exhibe ses charmes et ses enchantements dans toute leur somptuosité. La question de l'irrationalité de l'opéra est donc fondamentale. Dans le Neveu de Rameau, plus tardif cependant, mais centré sur Rameau et par conséquent sur des oeuvres qui relèvent de cette esthétique de la première moitié du XVIIIème siècle, l'opéra dès les premières pages, est présenté dans sa fascinante étrangeté baroque: 'Il y a de l'harmonie, des bouts de chants, des idées décousues, du fracas, des vols, des triomphes, des lances, des gloires, des murmures, des victoires à perte d'haleine; des airs de danse qui dureront éternellement'. ${ }^{1}$ Il est vraisembable que Diderot songe ici au Castor et Pollux de Rameau (cf. D.Heartz). Cependant ce recours au merveilleux et aux machines est un trait fondamental de tout l'opéra baroque, ce caractère composite également.

La théorie de l'opéra que l'on retrouve tant chez l'abbé Batteux que chez Voltaire répond donc bien à cette répartition des genres à laquelle se réfère la préface de Pellegrin que nous citions tout à l'heure. Dans ses Commentaires sur Corneille, à propos de la Toison d'or, Voltaire écrit: 'La partie fabuleuse de cette histoire semble beaucoup plus convenable à 
l'opéra qu'à la tragédie.' ${ }^{2}$ Même réflexion à propos de Médée: 'C'est à l'opéra, c'est à ce spectacle consacré aux fables, que ces enchantements conviennent ${ }^{\prime}{ }^{3}$ Reste à essayer de comprendre ce que signifie cet exil du merveilleux sur les terres de l'opéra. Et cela pose, d'une façon plus générale, la question du recours à la mythologie païenne au XVIIIème siècle, cette mythologie qui est si présente dans tous les arts. Ne serait-il pas plus rationnel d'utiliser une mythologie qui corresponde aux croyances du temps où l'on écrit? A l'époque d'Hippolyte et Aricie justement, des tentatives ont été faites d'utiliser la mythologie biblique. Dans deux oeuvres importantes en particulier: la Jephté de Montéclair et le Samson de Voltaire et Rameau. Mais cet opéra n'a jamais pu être joué, parce qu'il s'est heurté à la censure: elle supposait chez l'écrivain déjà fort connu une entreprise de dérision (ce qui n'était pas le cas pourtant) ou du moins une réinterprétation suspecte de la Bible vers le déisme (ce qui était exact). Les tentatives d'opéra biblique ou chrétien furent rares au XVIIIème siècle. Voilà donc ce 'merveilleux chrétien' que le Génie $d u$ Christianisme chantera, exclu de l'opéra. Ce qui a finalement des conséquences diverses: accroître l'irréalité et le merveilleux de ces dieux et de ces déesses - qu'invoquent aussi, la poésie et la peinture. Mais aussi laisser une plus grande liberté aux artistes. Liberté qui cependant n'est pas infinie car intervient alors un autre élément fondamental de l'esthétique classique: le respect des Anciens, à une époque où le public et en tout cas les critiques sont munis d'une forte culture antique. Ainsi l'abbé Pellegrin dans sa Préface se croit-il obligé de justifier son prologue en invoquant des textes grecs. Ce n'est pas chez Euripide qu'il trouve sa justification, mais chez d'autres auteurs moins célèbres: les librettistes ont le droit d'amalgamer plusieurs traditions, mais non d'innover totalement. Pour ce qui est, par delà la fantaisie, de donner, grâce à la musique, un surcroît de sens aux mythes antiques, les créateurs comme le public sont beaucoup plus libres en se mouvant ainsi dans une mythologie qui ne repose plus sur une croyance collective. La réflexion sur l'opéra serait donc à replacer dans une réflexion plus vaste et d'apparence plus sérieuse, parfois même érudite: la réflexion sur les fables et les mythes que poursuit le XVIIIème siècle et qui aboutira à la naissance de l'histoire des religions.

L'effort des Philosophes n'a-t-il pas consisté à ramener la philosophie et l'art sur la terre? Le troisième Entretien sur le fils naturel le proclame: 'Des hommes de génie ont ramené de nos jours la philosophie du monde intelligible dans le monde réel. Ne s'en trouvera-t-il pas qui rende le même service à la poésie lyrique, et qui la fasse descendre des régions enchantées sur la terre où nous habitons? ${ }^{\prime 4}$ Ce texte se situe plus tard dans le siècle, il n'en reflète pas moins un souci fondamental des Philosophes. Mais comment alors concilier cet effort de la philosophie et cette 
perpétuelle présence des dieux du théâtre lyrique? La discussion entre Dorval et Moi est intéressante parce que complexe et que, ici, comme souvent, on ne saurait ramener l'opinion de Diderot à celle de 'Moi': 'Moi: Quelle tragédie voulez-vous établir sur la scène lyrique? Dorval: L'ancienne. Moi: Pourquoi pas la tragédie domestique? Dorval: C'est que la tragédie, et en général toute composition destinée pour la scène lyrique, doit être mesurée; et que la tragédie domestique me semble exclure la versification. ${ }^{5}$ Voilà donc la question du réalisme étroitement liée à celle de la forme poétique. L'abbé Pellegrin avait sagement versifié l'histoire d'Hippolyte et Aricie, ce qui, nous l'avons vu, ne l'embarrassait guère, étant donnée sa grande fécondité. La tragédie en musique est écrite en règle générale à partir de livrets en vers. C'est dans l'opéracomique que la pratique est plus souple, et que la prose s'introduit largement. Faut-il donc établir une équivalence entre souci de la réalité et prose, ce que sembleraient montrer l'évolution du roman dans les voies du réalisme, et l'abandon peu à peu de la fantaisie du roman précieux? Ce n'est pas la versification qui exclut la vérité: la vérité des sentiments provient de l'intensité de la passion que le librettiste et le musicien sauront exprimer. L'opéra débarrassé de ses ornements parasites pourrait garder toute son intensité, qu'il soit en vers ou en prose. Et Diderot d'imaginer un opéra à partir de l'Iphigénie de Racine: 'L'état de Clytemnestre doit arracher de ses entrailles le cri de la nature; et le musicien le portera à mes oreilles, dans toutes ses nuances. ${ }^{6}$ Le poète et le musicien peuvent donner cette intensité de la passion sans qu'il n'y ait 'ni lance, ni victoire, ni tonnerre, ni vol, ni gloire'. ${ }^{7}$ La ressemblance de ce passage avec celui du Neveu de Rameau que nous citions précédemment est instructive: elle permet de penser qu'ici, c'est l'opéra ramiste que Diderot critique. Et l'on voit que le modèle auquel il se réfère est Racine.

En vers, ou en prose, peu importe, il s'agit de rendre le 'cri de la passion' et ainsi d'observer un autre dogme de l'esthétique classique, fortement revisité et réinterprété par le XVIIIème siècle: celui de l'imitation de la nature. Là aussi, la réflexion sur l'opéra est intéressante par le caractère limite, le caractère paradoxal de ce genre. En quoi le vol de Jupiter pour arbitrer entre les dieux, tel qu'on le voit dans Hippolyte et Aricie et ailleurs, peut-il 'imiter la nature'? Il semble plus facile de le justifier par l'imitation des Anciens. Mais puisque les Anciens ont imité la Nature ... . Le chant imite le cri de la passion, et les dieux sont susceptibles de passion. Peut-être est-ce aussi parce que l'anthropomorphisme est particulièrement manifeste dans la mythologie païenne qu'elle se prête mieux à l'opéra. Jupiter, Diane, l'Amour ont les passions des hommes et des femmes. 
Mais ce cri de la passion, qui est le langage même de la Nature, s'exprime-t-il aussi bien dans toutes les langues? Un des intérêts de la façon dont les Philosophes ont analysé l'opéra, c'est d'être intimement liée à leur réflexion sur le langage. On songe, bien entendu, aux textes fondamentaux de l'Essai sur l'origine des langues. Il n'est pas sûr que Rousseau l'eût écrit, en tout cas l'eût écrit sous cette forme, s'il n'avait pas été d'abord un musicien, s'il ne s'était pas lui aussi, et pendant toute sa vie, risqué sur la scène lyrique non seulement avec le fameux Devin $d u$ Village, mais plus tard encore avec Pygmalion. C'est ce désir de création lyrique qui sous-tend sa réflexion sur l'origine des langues: l'opéra parviendra-t-il à réintégrer ce langage premier qui n'était ni parole ni musique, qui était tout cela à la fois, dans 'l'unité de la mélodie'? Et à quelles conditions?

Nous se savons pas quel fut ce langage premier, mais toutes les langues actuelles ne sont pas également susceptibles de marcher sur ses traces. Si en 1733, la Querelle des Bouffons proprement dite n'a pas encore éclaté, le parallèle entre langue italienne et langue française à l'opéra est une constante au XVIIIème siècle que l'on retrouve déjà dans des textes des toutes premières années chez Raguenet, chez Le Cerf de la Viéville. L'opéra de Rameau, comme déjà celui de Lulli, ce musicien pourtant d'origine italienne, mais qui a souvent écrit sur les livrets de Quinault, semblerait apporter une preuve suffisante que la langue française peut être musicale. Pourtant les haines personnelles suscitées par la Querelle ont quelque peu obscurci l'objectivité des Philosophes. On le déplorera certes, et il fallut près de deux siècles pour que justice soit enfin rendue à Rameau. Mais ces parallèles entre les mérites de la langue française et de la langue italienne ont cependant l'intérêt d'aboutir à une réflexion sur les identités nationales. On regrettera aussi, bien entendu, que cette réflexion se limite à deux langues: l'opéra anglais de Purcell est inconnu des Philosophes, et Mozart n'a pas encore donné ses opéras allemands. Partisane, limitée, cette réflexion qui doit beaucoup à Montesquieu et qui se prolongera chez Stendhal, est pourtant un aspect important de la réflexion sur l'opéra, qui, par bien des aspects prépare la réflexion romantique, celle de Mme de Staël, en particulier, sur la spécificité des cultures européennes. 
La réception de l'opéra par les Philosophes met cependant en cause leur réflexion philosophique elle-même, et c'est là son intérêt majeur. Par delà l'analyse de tel aspect du spectacle, c'est le problème de la représentation du monde sur la scène lyrique et du sens de cette représentation qu'ils se posent.

Nous avons vu en effet quelle tension s'établissait chez les Philosophes entre une passion de l'opéra vécue quotidiennement, et cette exigence de rationalité dont ce genre semble s'éloigner si délibérément. Cette victoire momentanée de la magie du spectacle sur les exigences de la rationalité, elle est confessée, tout naïvement dans l'article 'Opéra' de l'Encyclopédie: “Ce que l'art (du musicien) ajoute à l'art du poète supplée au manque de vraisemblance qu'on trouve dans ces acteurs qui traitent leurs passions, leurs querelles, et leurs intérêts en chantant, puisqu'il est vrai que la peine et le plaisir, la joie et la tristesse s'annoncent toujours ici par des chants et des danses; mais la musique a tant d'empire sur nous, que ses expressions commandent à l'esprit, et lui font la loi'.

Certaines solutions s'esquissent cependant. Et d'abord celle de simplifier l'opéra baroque, en le faisant se rapprocher de la tragédie antique, telle que les hommes du XVIIIème siècle peuvent se la représenter. Ils savent en effet que les tragédies de Sophocle et d'Euripide étaient soutenues par la musique, et ils se représentent la diction de ces textes à l'image des récitatifs de l'opéra, ce qui n'est probablement pas faux, malgré le manque de documentation proprement musicale: ils ne connaissent guère la musique ancienne que par les traités sur la musique que nous a laissés l'Antiquité, d'où d'ailleurs la facilité avec laquelle cette musique antique devient mythique. Puisque se rapprocher des Anciens, $c^{\prime}$ est aussi se rapprocher de la nature, c'est dans un même mouvement que les écrivains réclament à la fois de se rapprocher du vraisemblable et des modèles laissés par la tragédie antique. Cette exigence de simplicité qui leur semble synonyme d'une approche de la réalité aboutit à de grandes transformations du genre. De même que Diderot préconise le drame bourgeois, il préconise aussi dans les Entretiens sur le Fils Naturel un opéra qui serait un opéra bourgeois, l'opéra-comique, qui tendrait vers le tragique, sinon les larmes, dans ce mélange des genres qu'il réclame bien avant la bataille d'Hernani. Soucieux de rester sur le registre purement tragique, Gluck dans sa réforme de l'opéra s'efforcera de retrouver la simplicité antique. Mais l'opéra du XIXème siècle saura tirer parti de ces idées lancées par Diderot, et dont la Carmen de Bizet serait un bel accomplissement.

La conception de la danse dans l'opéra est modifiée en profondeur par cette réflexion des Lumières. Les ballets ne devront plus être de 
simples divertissements; ils devront être intégrés étroitement dans l'action, présenter, autant que faire se peut, une sorte de caractère de nécessité. La technique de la danse elle-même va changer profondément avec la révolution qu'amène Noverre, révolution si chaudement applaudie par Diderot. Cette danse d'expression qui se rapproche de la pantomime, s'écarte du caractère purement décoratif des ballets de l'opéra. Mais il n'est que juste de noter que, si révolutionnaire que soit Noverre, ce souci d'intégrer étroitement les ballets à l'action était déjà présent chez Rameau, et cela dès Hippolyte et Aricie.

Simplifier l'opéra, certes, mais aussi le charger d'un sens. Et la tentative de Voltaire dans Samson mérite de retenir l'attention. Car il propose à la fois une simplification des formes, il veut que l'opéra cesse de s'affadir dans les méandres de la galanterie, et symboliquement, réduit le rôle de Dalila; mais surtout il veut inaugurer ainsi l'opéra philosophique. Entreprise hardie: Samson devient un soldat des Lumières. On se demandera cependant si l'entreprise était viable. L'obstacle essentiel n'était peut-être pas l'opposition de la censure, mais la difficulté de créer un opéra à thèse. 'L'autorité fictive,' pour reprendre le titre du beau livre de Susan Suleiman, pose déjà des problèmes esthétiques; mais l'opéra autoritaire est-il possible? En tout cas le XVIIIème siècle n'en a pas laissé d'exemples vraiment convaincants.

On voit que la question est plus complexe: il ne suffit pas de renoncer au merveilleux, pour que la représentation du réel prenne un sens. Le réel a-t-il une signification, et la représentation du réel par l'art en acquiert-elle un, par le fait même qu'elle est représentation? Ne faut-il pas pour découvrir ce sens réintroduire le mythe? L'article "Opéra" de l'Encyclopédie bâtit en effet un mythe qui fait songer à ceux de Platon, ou peut-être davantage de Marivaux: 'Supposons pour un moment que le roi de France envoyât les acteurs et les actrices de l'opéra peupler une colonie déserte, et qu'il leur ordonnnât de ne se demander les choses les plus nécessaires et de ne converser ensemble que comme ils parlent sur le théâtre; les enfants qui naîtraient au bout de quelque temps dans cette île bégayeraient des airs, et toutes les inflexions de leur voix seraient mesurées. Les fils des danseurs marcheraient toujours en cadence, pour se rendre en quelque lieu que ce fût ... si les peuples sauvages voisins de l'île où dans ma supposition j'ai relégué l'opéra venaient à ce spectacle, loin de le trouver ridicule, je ne doute guère qu'ils n'admirassent le génie des acteurs, et qu'ils ne les regardassent comme des intelligences célestes'. Ce mythe est inventé par Cahusac pour justifier l'opéra dans sa spécificité irréductible. L'opéra est un monde en lui-même. Mais s'il était l'image du monde? Et si la part d'irrationalité qu'il contient n'était autre chose, en définitive, que la représentation de tout ce qui dans l'univers échappe encore, échappera peut-être toujours à l'emprise de la 
Raison. C'est à Diderot qu'il faut revenir et à La Promenade du sceptique qu'on se référera. Le déiste naîf, explique-t-il, pense que tout dans le monde a été fait pour lui, tel le provincial qui arrive à l'Opéra: 'c'est la présomption du provincial nouvellement débarqué, qui croit que c'est pour lui que Servandoni a dessiné les jardins d'Armide, ou construit le palais du Soleil. Nous avons devant nous une machine inconnue'. ${ }^{8}$ Cette naïveté du déiste guette aussi le matérialiste qui croirait pouvoir tout comprendre de cette 'machine inconnue'. Ces machines de l'opéra dont l'usage immodéré a tant été blâmé comme le symbole même de la pure fantaisie du merveilleux, acquièrent donc ici un sens métaphorique. L'irrationalité de l'opéra, qui n'est peut-être que l'expression la plus magnifique de tout ce qui dans la musique et dans l'art échappe à la Raison, ne deviendrait-elle pas aussi une façon de représenter le réel? tout ce qui dans le réel dépasse encore l'emprise de la Raison humaine? Loin que l'irrationalité de l'opéra constitue un écart de la réalité et une perte de sens, elle devient alors un sûr moyen de faire pressentir à l'homme qu'il peut $y$ avoir du sens même là où sa Raison ne s'aventure qu'avec une certaine prudence, dans ce domaine, par exemple, des sciences de la vie que le XVIIIème siècle ne fait que pressentir. L'opéra pour les Philosophes est bien le Théâtre du Monde.

\section{BÉATRICE DIDIER}

École Normale Supérieure

\section{Endnotes}

1 Le Neveu de Rameau, ed. Jean Fabre (Genève: Droz, 1950) 6.

2 Commentaires sur Corneille, ed. David Williams. Oeuvres complètes (Banbury: Voltaire Foundation, 1975) 55:823.

3 Oeuvres complètes 54:31.

4 Oeuvres complètes (Paris: Le Club français du livre, 1970) 3:197-8.

5 Ibid., 203.

6 Ibid., 205.

7 Ibid., 207.

8 Diderot, Oeuvres complètes 1:370. 